\title{
Proyecto sartre. ¿una conducción autónoma o reducción de la contaminación ambiental?
}

\section{Sartre project. An autonomous driving or reduction of environmental pollution?}

Luis Xavier Orbea Hinojosa

Universidad Tecnológica Equinoccial, Ecuador

Edwin Giovanny Puente Moromenacho

Universidad Internacional del Ecuador, Ecuador

Cristhian Alexander Guano Calvache

Universidad Tecnológica Equinoccial, Ecuador

Marco Vinicio Noroña Merchán

Universidad Internacional del Ecuador, Ecuador

Autor para correspondencia: luis.orbea@ute.edu.ec; manoroname@uide.edu.ec

Fecha de recepción: 25 de Febrero de 2018 - Fecha de aceptación: 25 de Junio de 2018

Resumen: En la actualidad no es novedad el poder hablar sobre vehículos con sistemas de conducción autónoma. Hoy en día el campo automotriz en sus diferentes marcas de vehículos a desarrollado diferentes niveles y formas de conducción autónoma que van desde la necesidad (con conductor) hasta la no necesidad de control del vehículo (conductor que limite a que el vehículo cumpla con sus necesidades). Es decir, la conducción desde el nivel 0 hasta la conducción realizada mediante dispositivos electrónicos como el teléfono celular. La compañía automotriz Volvo en el año 2011 presento el Proyecto "SARTRE" (SAfe Road TRain for the Environment - Trenes de Carretera Seguros para el Medio Ambiente) la cual pretende que sin cambios de infraestructura en las vías y con mínimas modificaciones en los actuales modelos de vehículos se los dote de avanzados sistemas de seguridad para lograr una conducción segura y eficiente. En el presente artículo se analizará si el Proyecto SARTRE es una manera más hacia la conducción autónoma o se enfoca a la reducción de la contaminación ambiental.

Palabras Claves: conducción autónoma; contaminación; seguridad; tecnología; vehículo Abstract: At present, it is not new to talk about vehicles with autonomous driving systems. Today the automotive field in their different brands of vehicles developed different levels and forms of autonomous driving ranging from the need (with driver) to no need for control of the vehicle (the driver that limits the vehicle meets your needs). That is to say, driving from level 0 to driving through electronic devices such as a cell phone. The automotive company Volvo in 2011 presented the "SARTRE" Project (SAfe Road TRain for the Environment) which aims that without infrastructure changes in the roads and with minimal modifications in the current models of vehicles are endowed with advanced safety systems to achieve safe and efficient driving. In this article we will analyze whether the SARTRE Project is a way to autonomous driving or focused on the reduction of environmental pollution.

Key Words: autonomous driving; contamination; security; technology; vehicle 


\section{Introducción}

La necesidad de siempre innovar, mejorar y desarrollar tecnologías que permitan la seguridad, el confort y la reducción de la contaminación ambiental en el campo automotriz ha transcendido de forma significativa. Cada tecnología ha tenido siempre un propósito específico en el vehículo, por ejemplo los airbags diseñados para proteger al o los ocupantes del vehículo en caso de una colisión, el sistema Active Cruise Control con función Stop \& Go que es un programador de velocidad con control adaptativo, el Active Line Assist (asistente de línea activo) para permanecer dentro del carril, el Line Assist con la doble función de asistente de cambio de carril y detección de obstáculos en los ángulos muertos, entre otros sistemas similares. (Prieto, 2012).

La compañía automotriz Volvo en su línea de prototipos de camiones, presentó en el año 2011 el Proyecto SARTRE, que consiste en que el convoy líder a través de un sistema de comunicación inalámbrica permita que varios vehículos se "acoplen" a él, y que los vehículos "acoplados" se conduzcan de forma autónoma permitiendo que el conductor pueda descansar, o realizar diferentes tareas (ver películas, comer, leer o cualquier otra cosa distinta a la conducción) con el fin de reducir la contaminación ambiental, aumentar la seguridad vial, ahorrar combustible y el aumentar el confort de los ocupantes.

Ahora bien, si se analiza este proyecto de Volvo es una forma más de conducción eficiente por lo cual deja muchos espacios para decir si realmente es una conducción autónoma. Dado este preámbulo se define que el objetivo principal de este estudio es analizar si el Proyecto SARTRE de Volvo es parte de la conducción autónoma o solo un sistema que permite la reducción la contaminación ambiental, para ello se dará a conocer las generalidades del proyecto SARTRE, así como también sus ventajas y desventajas, y la tecnología que conlleva.

\section{Materiales y métodos}

\section{Generalidades}

SARTRE o Safe Road Trains for the Environment, es un proyecto financiado por la Unión Europea y desarrollado por la Compañía Volvo cuyo objetivo es desarrollar la tecnología necesaria para viajar en convoy y permitir que los vehículos anclados ahorren combustible, se reduzca la emisión de gases del vehículo y exista mayor confort en la conducción. (Artes, 2011)

El concepto es sencillo, un vehículo pesado, ya sea un camión o autobús conducido por un conductor profesional es seguido por un pelotón de vehículos cuyos conductores han cedido los mandos a un sistema de seguimiento. Cada vehículo permanece a una distancia reducida de su predecesor, que se mantiene constante. Una vez que un auto se convierte en seguidor, el conductor de este puede dejar de conducir, dado que una serie de sensores de video, radar y tecnología específica se encargarán de mantener la distancia con el vehículo líder y siguiendo la misma trayectoria. En cualquier momento puede sumarse otro vehículo seguidor al convoy. El conductor de cualquiera de los autos seguidores puede abandonar el "tren de carretera" en cualquier momento, ya sea cambiándose de carril o tomando una salida de la autopista, en ese 
caso, el espacio abandonado se reducirá paulatinamente y el tren adquirirá una nueva configuración, con un seguidor menos.

\section{Tecnología}

Todo vehículo autónomo se compone de un conjunto de sistemas y sensores que permiten no solo que el vehículo adquiera "vida propia", sino que también la persona detrás del volante sea un controlador y más no solo un espectador (Frigati, 2012). En el caso de SARTRE todo se basa en la comunicación entre vehículos y para ello necesita de:

\section{Radar y Lidar (radar láser)}

Se combinan para aumentar el conocimiento del entorno y actualizar el mapa de su entorno para evitar obstáculos; recogiendo datos de forma periódica para el proceso de localización, mapeo del entorno y detección de obstáculos que se produce continuamente. El medidor laser escanea el entorno haciendo sucesivas pasadas y calcula la distancia a los objetos cercanos en base al tiempo que el haz de luz tarda en volver, creando un modelo tridimensional del entorno.

\section{Sensor de ultrasonidos (Figura 1)}

Para eliminar ruido innecesario y que pueda alterar la ruta.

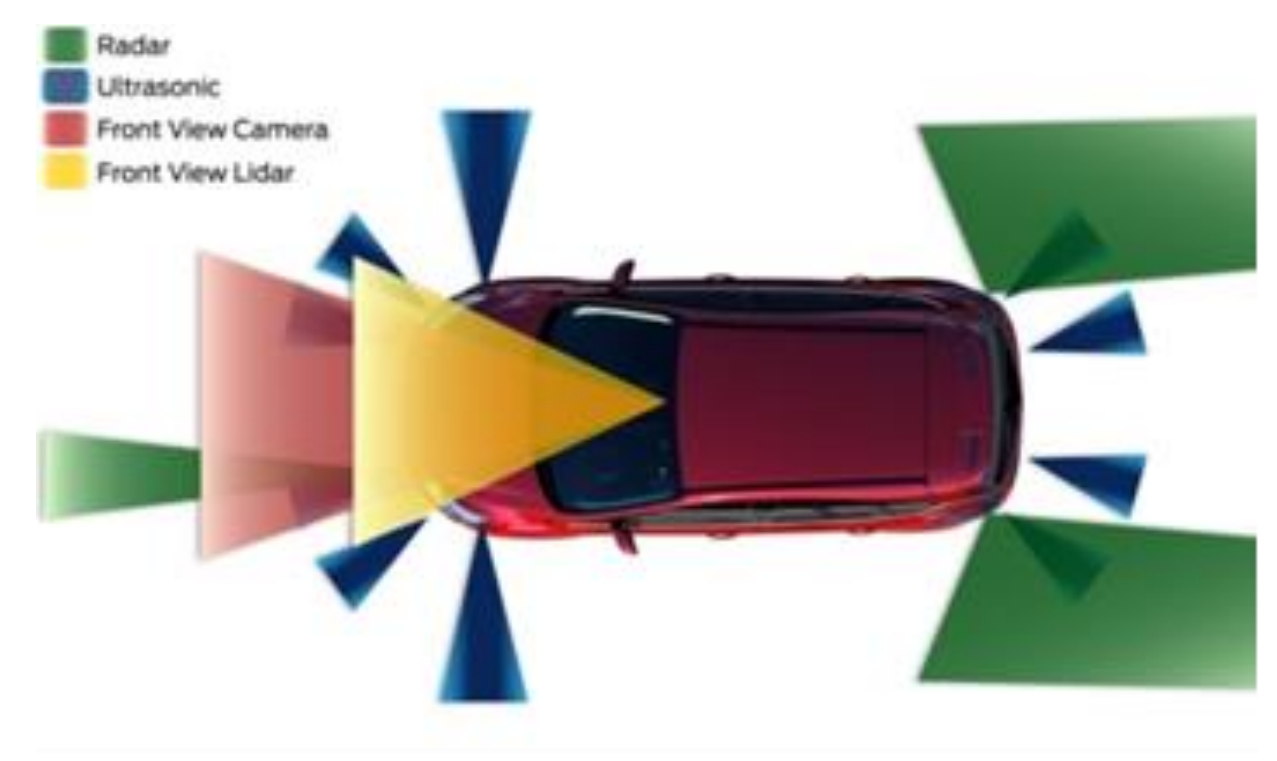

Figura 1. Posición y zona de trabajo de los radares y del sensor de ultrasonido. (Frigati, 2012)

\section{Cámaras de vídeo (Figura 2)}

El vehículo convoy de poder detectar obstáculos en el frente, la proximidad de un vehículo que va a rebasar por la parte posterior, los vehículos que le siguen, entre otros. 


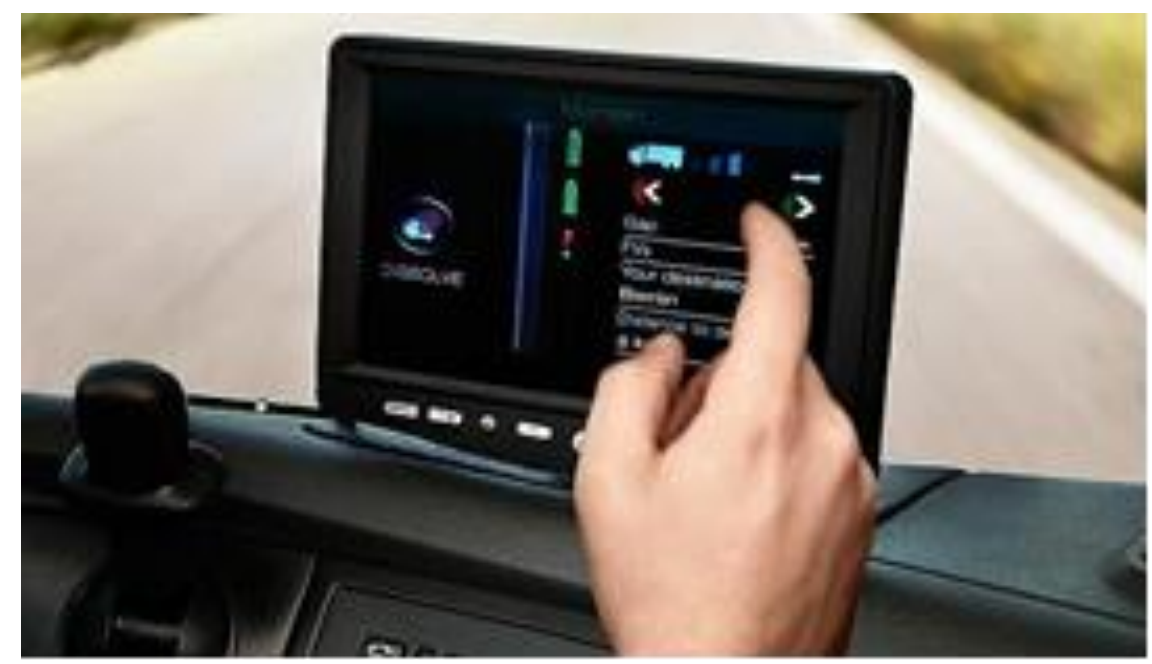

Figura 2. Pantalla que permite conocer el lugar de destino del camión líder, así como también el poder anclarse a él (Frigati, 2012)

\section{Sistemas de comunicación inalámbrica entre vehículos y el mismo vehículo (Figura 3)}

Se combina con las cámaras de video para obtener una situación real del tráfico. La autocomunicación sirve en caso colisión, el abandono de uno de sus vehículos seguidores (en el caso del convoy), la detención total o parcial de algún vehículo que se encuentra en la hilera, entre otros.

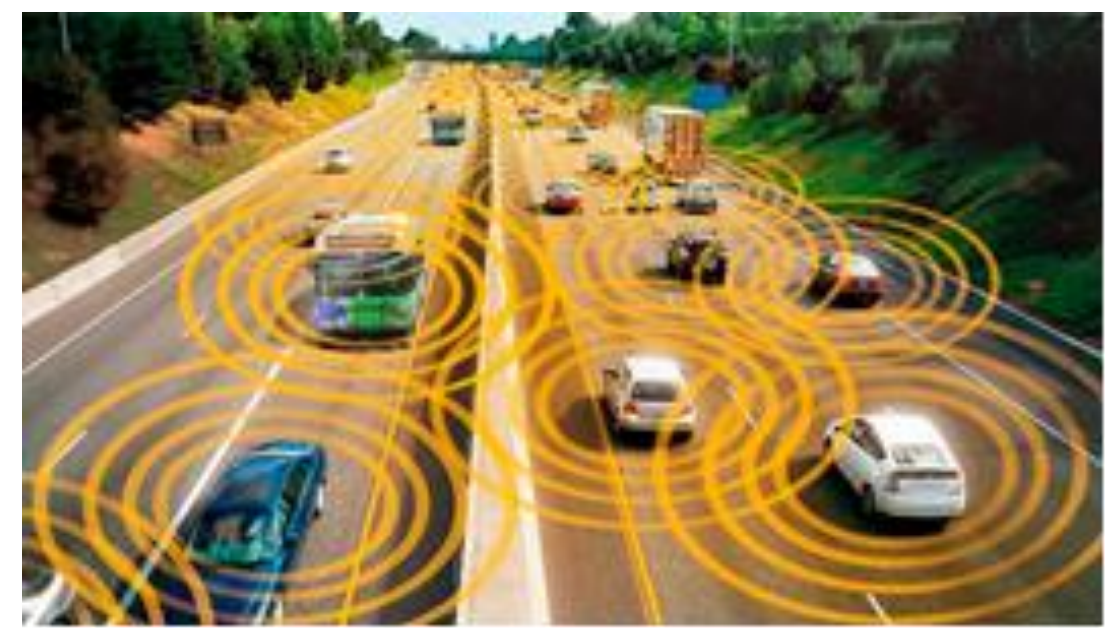

Figura 3. Sistema de comunicación inalámbrica (Gonzales, 2014)

\section{GPS (Figura 4)}

Utiliza un sistema de navegación inercial (INS - Inertial Navigation System), puesto que el GPS no es preciso en diversos metros debido a un retraso en la emisión. INS usa acelerómetros y sensores de rotación para calcular continuamente una estimación de la posición, orientación y velocidad del movimiento del vehículo, combinándolo con la posición del GPS se obtiene una posición con mayor exactitud (Ibañez, 2012) 


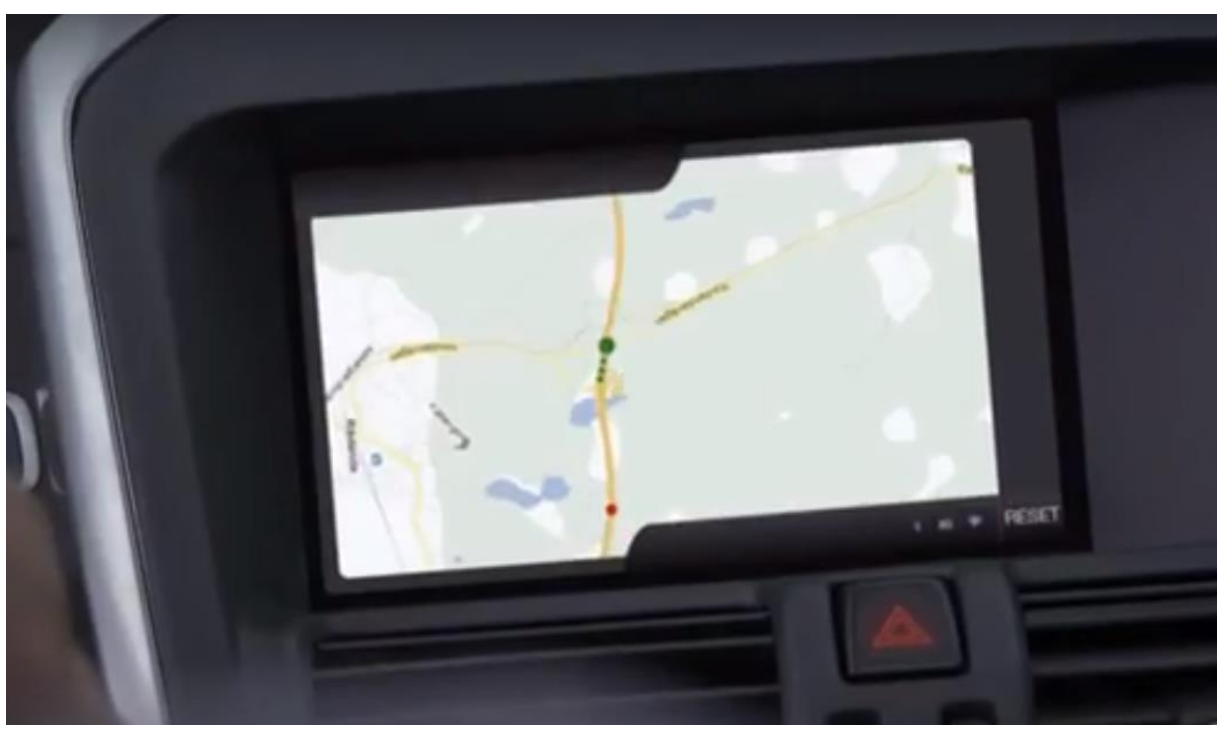

Figura 4. Sistema de navegación inercial (INS) (Ibañez, 2012)

\section{Resultados y Discusión}

Si a este proyecto lo ubicamos dentro de los niveles de conducción autónoma sería en el Nivel 2 (automatización parcial) porque el vehículo que va en la hilera es capaz de moverse de forma independiente en ciertos escenarios. (El Comercio, 2015)

Entre las principales ventajas tenemos:

- Reducción de los accidentes

- Mejora de la fluidez del tráfico

- Ahorro de combustible.

- El conductor dispondrá de tiempo para realizar otras tareas que no sean la conducción en sí, como leer un libro, comer, hablar por teléfono, entre otros.

- Se promueve el transporte sostenible y eficiente.

- El impacto medioambiental se ve reducido debido a que los vehículos circulan el uno detrás del otro reduciendo así la resistencia al aire.

- Se calcula que el ahorro energético será del 20\%. (TECNALIA, 2012)

Entre las principales desventajas tenemos:

- La inteligencia artificial.

- Costos de producción del vehículo tanto del que será guía como de los seguidores.

- Las plazas de trabajo que giran alrededor del campo automotriz (seguros, concesionarios, talleres de mecánica, entre otros).

- La convivencia entre vehículos autónomos y los vehículos convencionales. (Admi. Corporation, 2017)

- Perdida de privacidad total, puesto que el vehículo necesita satélites en tiempo real para funcionar. (Gonzales, 2014) 
De acuerdo con las ventajas y desventajas obtenidas se puede concluir que el proyecto SARTRE si puede ser una forma más de conducción autónoma pero que su principal contribución es hacia la reducción de gases contaminantes, la seguridad vial y el confort de los conductores.Un resultado que se puede añadir es que actualmente el Proyecto SARTRE permite trenes de carretera con hasta 8 vehículos conectados de forma inalámbrica haciendo uso de la conducción autónoma. Dicho resultado se obtuvo en el mes de septiembre de 2012. (Clavero, 2012)

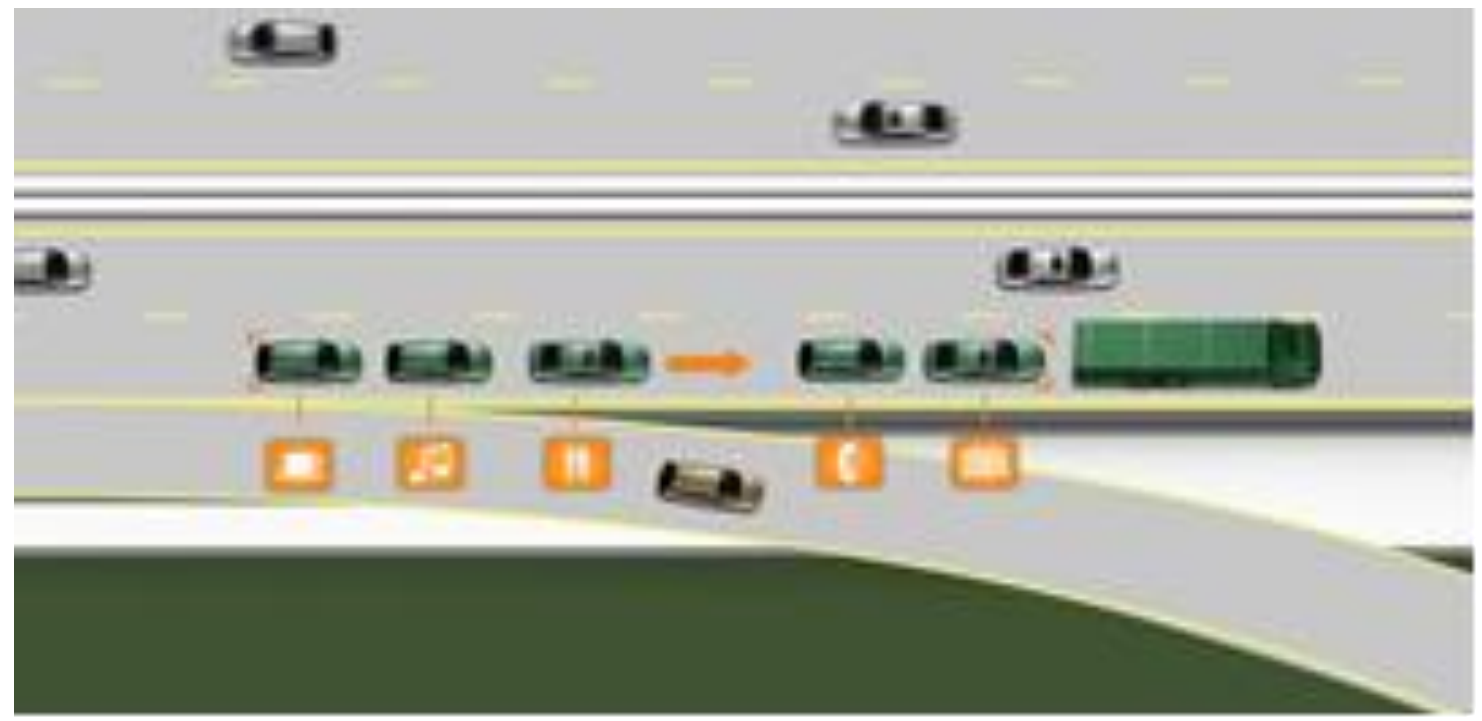

Figura 5. Proyecto SARTRE (Clavero, 2012)

\section{Conclusiones}

Un vehículo autónomo puede contribuir a una conducción eficiente, pero a la vez crea un debate mundial en cuanto a la inteligencia artificial, por cuanto el humano se vuelve un ser dependiente de la tecnología. Para garantizar la sostenibilidad del transporte por carretera, debemos sustituir a la mayor brevedad los combustibles fósiles por nuevos combustibles y sistemas de propulsión, más eficientes y menos contaminantes.

No hay combustibles fósiles para atender el aumento de la demanda que provocará la motorización de los países emergentes. Se espera que en 2050 habrá más de 2.000 millones de vehículos circulando. El parque automovilístico chino puede crecer en tasas anuales superiores al $20 \%$.

\section{Bibliografía}

Acelerando. (12 de 09 de 2012). Acelerando Transporte. Obtenido de Gasolina Eco-país reemplazará a Extra hasta el 2017: http://www.acelerando.com.ec/industria/mundoverde/443-gasolina-eco-pais-reemplazara-a-extra-hasta-el-2017 
Admi. Corporation. (10 de 06 de 2017). Coches sin conductor. Obtenido de http://www.cochesinconductor.com/2017/06/que-desventajas-tendran-los-cochesautonomos/

Artes, D. (26 de 07 de 2011). DiarioMotor. Obtenido de https://www.diariomotor.com/tecmovia/2011/07/26/proyecto-sartre-una-forma-realistade-reducir-el-consumo-incrementar-la-seguridad-y-mucho-mas/

Clavero, D. (07 de 12 de 2012). DiarioMotor. Obtenido de https://www.diariomotor.com/tecmovia/2012/12/07/volvo-trucks-ve-posible-la-llegadade-los-trenes-de-carretera-inteligentes-en-10-anos/

El Comercio. (5 de 06 de 2015). El Comercio. Obtenido de http://www.elcomercio.com/deportes/carburando-autos-automoviles-autonomosventajas.html

Frigati, M. (17 de 02 de 2012). Hoy.digital. Obtenido de http://hoy.com.do/proyecto-deconduccion-autonoma-sartre/

Gonzales, G. (26 de 10 de 2014). Thinkbig. Obtenido de https://blogthinkbig.com/ventajas-cochesautonomos

Ibañez, N. (10 de 08 de 2012). Xataka. Obtenido de https://www.xataka.com/automovil/tecnologia-para-el-coche-avances-en-conduccionautonoma

INEN. (2002). Gestión Ambiental, Aire, Vehículos Automotores. Quito: INEN.

Martínez, E., \& Díaz de Mera Morales, Y. (2014). Contaminación atmosférica. Universidad de Castilla-La Mancha, 13. Obtenido de Contaminación atmosférica.

Prieto, F. (10 de 08 de 2012). ABC.es. Obtenido de http://www.abc.es/20121008/motorreportajes/abci-proyecto-sartre-conduccion-autonoma-201210071011.html

TECNALIA. (03 de 02 de 2012). Tecnalia. Obtenido de https://www.tecnalia.com/es/industriatransporte/noticias/proyecto-sartre-conduccion-autonoma.htm

Tecnum. (2002). Ciencias de la tierra, Sustancias que contaminan la atmósfera. Gipuzkoa: Tecnun.

The Internatioanal Council On Clean Transportation. (2011). Introducción A La Refinación Del Petróleo Y Producción De Gasolina Y Diésel Con Contenido Ultra Bajo De Azufre. Theicct, 1-45. 
Vanegas, E. (01 de Marzo de 2016). Informe Proyecto ECOPAÍS-01-03-16-1. Obtenido de Lineamientos principales del proyecto EcoPaís: https://es.scribd.com/document/357908420/Informe-Proyecto-Ecopais-01-03-16-1 